Meta

Journal des traducteurs

Translators' Journal

\title{
Sémantique et traduction : exercices
}

\section{Jean-Claude Choul}

Volume 25, numéro 2, juin 1980

URI : https://id.erudit.org/iderudit/002237ar

DOI : https://doi.org/10.7202/002237ar

Aller au sommaire du numéro

Éditeur(s)

Les Presses de l'Université de Montréal

ISSN

0026-0452 (imprimé)

1492-1421 (numérique)

Découvrir la revue

Citer cet article

Choul, J.-C. (1980). Sémantique et traduction : exercices. Meta, 25(2), 230-243.

https://doi.org/10.7202/002237ar d'utilisation que vous pouvez consulter en ligne.

https://apropos.erudit.org/fr/usagers/politique-dutilisation/ 


\section{Sémantique et traduction : exercices}

Jean-Claude Choul

Sous ce titre quelque peu général nous présentons quelques exercices dont le traducteur pourrait tirer parti dans l'apprentissage et dans la pratique de sa discipline. Il s'agit donc d'exercices d'apprentissage et d'assouplissement qui se présentent également comme compléments aux exercices terminologiques ${ }^{1}$. Ils répondent en particulier aux problèmes de lecture du traducteur, plus spécialement dans la consultation des dictionnaires, c'est-à-dire lorsqu'un choix devient délicat (parmi plusieurs acceptions) ou lorsqu'il semble nécessaire de procéder à une extension de sens, ou encore de forger sinon un terme du moins un syntagme dénominatif.

Les exercices s'appuient d'une part sur le comportement extérieur du traducteur dans l'exercice de sa spécialité et de l'autre sur les apports possibles de la sémantique linguistique à la traduction.

\section{Qu'est-ce qu'un traducteur?}

Par comportement extérieur, il faut entendre ce que fait un traducteur lorsqu'il est aux prises avec un texte lui réservant quelques difficultés (quel qu'en soit l'ordre). S'il ne comprend pas la phrase qu'il a sous les yeux, il a généralement recours à un dictionnaire de langue (anglaise) où il vérifiera le ou les termes qui lui semblent être à l'origine de sa difficulté de compréhension. Le traducteur est donc, en gros, un manipulateur de livres. Les exercices qui suivent auront ainsi pour objet la manipulation de sources écrites (ou d'autres supports analogues).

\section{Compréhension et sémantique}

S'il découvre le terme dans le dictionnaire, il s'efforce alors d'apparier la définition ou l'une des acceptions (cas de polysémie) à la position occupée par le terme dans la phrase en question. Cet effort est répété pour chaque terme litigieux. Il s'agit d'un contrôle de compréhension. Cette opération peut porter le nom de sémantisation, en particulier si l'on songe aux cas, relativement nombreux, où le traducteur se trouve devant un quasi blanc: terme ou forme non

1. Voir Dubuc (1978). 
répertorié qui doit pourtant recevoir (se voir assigner) un sens. La sémantisation peut alors se faire par le contexte auquel on emprunte des traits pour les faire figurer dans une éventuelle description du terme. Il peut aussi bien s'agir d'une dérivation : comment interpréter locational pattern of development sinon à partir de location ? La nécessité d'une sémantisation absolue (interprétation originale) peut aussi se présenter, mais le contrôle par les répertoires doit précéder toute autre démarche, pour éviter par exemple une interprétation poétique de: la mouette est un voilier très résistant (le contrôle peut se faire dans le Petit Larousse).

Recherche de l'équivalence

Toujours en suivant le traducteur, on notera qu'en guise d'étape subséquente, comme il dispose du sens des termes et qu'il est parvenu à une combinaison satisfaisante des acceptions pour se constituer le «sens de la phrase», il cherchera à transposer ce sens dans la langue d'arrivée. Cette recherche de l'équivalence semble se fonder (tout comme la compréhension) sur la paraphrase, notion que nous retrouverons plus loin. Si l'on se fie à son comportement extérieur en cas de difficulté, le traducteur, manipulateur de livres, consulte alors un répertoire bilingue, dictionnaire, lexique, etc. Pressé, il pourrait se contenter d'organiser en phrase les sens retenus, mais on ne saurait trop le mettre en garde. Une troisième étape s'impose, également illustrée par son comportement. S'il a choisi géographique comme équivalent de locational, que fait-il?

\section{Équivalence}

En cas de doute le traducteur consultera généralement un dictionnaire de langue (française), Lexis, Petit Robert ou autre. S'il n'est pas satisfait, rien ne l'empêche d'aller jusqu'au Trésor. Cette étape s'intitulerait : contrôle de l'équivalence. Elle se fera sur deux modes, qui peuvent également exister dans l'étape de contrôle de la compréhension.

\section{Syntagmatique et phraséologie}

Le mode syntagmatique du contrôle a pour objet de vérifier le sens d'un terme en syntagme, c'est-à-dire plus exactement dans la construction qui conditionne ce sens et consiste à identifier la sémiotaxie (l'assemblage sémantique) qui correspond au sens que l'on cherche à vérifier ${ }^{2}$. Ce mode de contrôle est particulièrement efficace et pertinent dans le cas des verbes. Le mode phraséologique par contre (bien qu'il soit complémentaire du premier et souvent associé dans la pratique) portera sur la distribution du terme, sa collocation, et répond à la question : à quel terme puis-je associer un terme donné ? Ce contrôle peut permettre de combler une lacune provisoire dans la recherche de l'équivalence. Si l'on a retenu géographique pour locational dans l'exemple ci-dessus, doit-on se contenter de modèle, structure, etc. (considérés comme insatisfaisants) ou

2. Sur la sémiotaxie, voir Choul (1979). 
admettre répartition qui présente une certaine cohésion avec géographique en particulier si l'on conserve à development son trait de mouvement.

Grosso modo on peut donc isoler trois étapes dans le comportement du traducteur : contrôle de compréhension, recherche de l'équivalence et contrôle de l'équivalence (de l'expression), et imaginer un schéma provisoire et elliptique $^{3}$ :

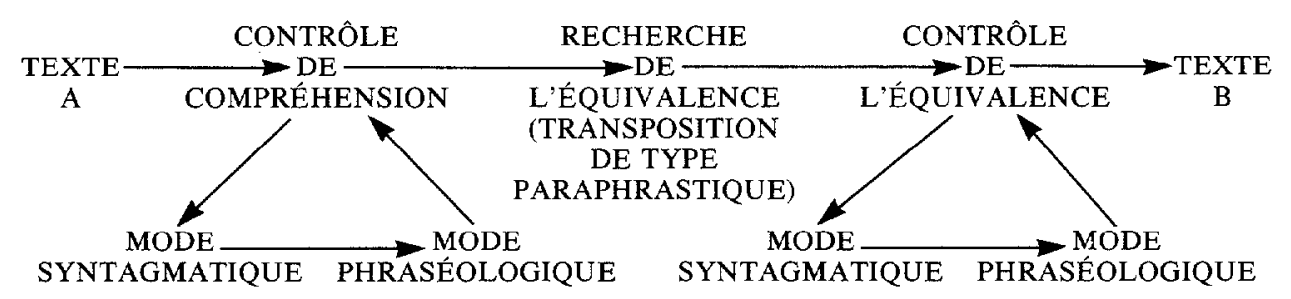

Les exercices proposés se présentent comme des illustrations des trois étapes d'une part et de l'autre comme des moyens d'assurer un déroulement plus efficace des opérations - tout en constituant, dans un premier temps, ou, d'un point de vue théorique ou méthodologique, un moyen d'étudier la contextualisation.

\section{Fiches syntagmatiques}

Le mode syntagmatique, série d'opérations d'appariement d'un sens à une forme, aura comme exercice la forme d'une fiche.

La préparation et la rédaction des fiches a été proposée à des étudiants de $3^{\mathrm{e}}$ et de $4^{\mathrm{e}}$ année du B.Sp. en traduction de l'Université d'Ottawa. L'expérience en cours démontre dès l'abord a) la nécessité de ce genre de contrainte, b) l'intérêt méthodologique des recherches.

La nécessité est surtout illustrée par le fait que les traducteurs ne semblent pas avoir d'idée très précise sur la nature du sens, alors que c'est justement le sens qui fait l'objet du transfert réalisé par l'acte de traduction ${ }^{4}$.

L'intérêt méthodologique devient évident si l'on songe que les manipulations proposées permettent d'approfondir la connaissance a) de la langue et de ses mécanismes, b) du monde (encyclopédique), c) des supports exploités: évaluation et adéquation des sources à des tâches particulières. On peut par exemple s'apercevoir que le Petit Larousse constitue une bonne base pour la langue technique, que le Bordas du français vivant sensibilise à la question de l'idiomaticité, etc.

3. Il s'agit toujours du comportement extérieur, et non des aspects psycholinguistiques de la traduction.

4. Pour être juste, il faut signaler que ni linguistes ni sémanticiens ne s'entendent sur le sens, mais le traducteur devrait tenter de systématiser cette opération sémantique intuitive qu'est la paraphrase, cf. Greimas (1979); sur la difficulté de cerner le sens, voir entre autres Lyons (1977) et Dillon (1977), et pour un exposé très clair de ce qu'est le sens par syntagmation, voir Benveniste (1976). 
Le terme même de fiche est une illustration des mécanismes qu'elle se propose de mettre en évidence. En effet, il s'agit moins du support que d'un syntagme en voie de lexicalisation, sur le modèle de fiche technique.

Analyse

Il semble audacieux de vouloir analyser le syntagme alors que les lexèmes se prêtent déjà difficilement à une analyse sémique (décomposition en traits sémantiques) exhaustive. En fait, une perspective syntagmatique s'interdit de clore absolument la fiche sémique (la liste plus ou moins ordonnée) des sèmes d'un lexème. L'analyse du syntagne est donc bornée. Plus exactement, dans la fiche syntagmatique, elle se limite à faire figurer l'équivalence intralinguistique (synonymie ou paraphrase) d'une suite de syntagmes donnés (partant d'un terme-souche).

Il s'agit donc d'une analyse dynamique des variations en contexte proche, de l'étude des processus sémantiques à l'œuvre dans le syntagme, mais qui devrait permettre également par l'objet même de son analyse d'isoler le ou les sens de groupe de mots apparentés.

\section{Lexico-sémantique}

On constate aussitôt que la méthode peut recouper le principe de définition adopté par les lexicographes modernes (dont LEX, BOR), et qui consiste à «replacer le mot dans sa construction». La fiche syntagmatique devrait avoir pour objet premier la mise en évidence des rapports entre construction et sens (syntaxe et sémantique, éventuellement) ${ }^{5}$.

En fait l'hypothèse sous-jacente à la méthode pose que le sens est le propre du syntagme (ce qui ne revient cependant pas tout à fait à dire que le sens est syntaxique, comme l'a fait Guiraud ${ }^{6}$ ). Le sens n'existe donc pas avant la syntagmation. Il n'y a que sémantisme, dans lequel les termes du syntagme du fait de leur mise en présence opèrent un tri. Le sémantisme, plutôt que de correspondre à la polysémie, s'identifierait à ce que regroupent les entrées des dictionnaires ou encore à ce qu'un locuteur peut dire d'un terme.

LEX (1977) fournit un excellent exemple de ce tri, tout comme DDL

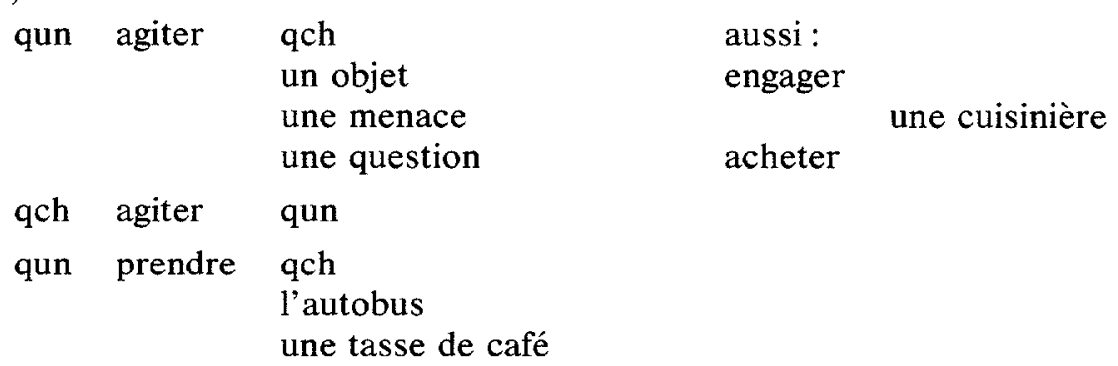

5. Sur les constructions à partir desquelles il est possible de travailler, voir Le Goffic et Combe McBride (1975).

6. Guiraud (1970). 
On note ainsi que l'occupation des positions qun-qch ou encore l'insertion de particules vont spécialiser le sens jusqu'à en empêcher la lecture directe (transparente) : la fiche syntagmatique a toutes les chances de se terminer par une ou plusieurs locutions, comme jouer, par exemple :

TABLEAU I

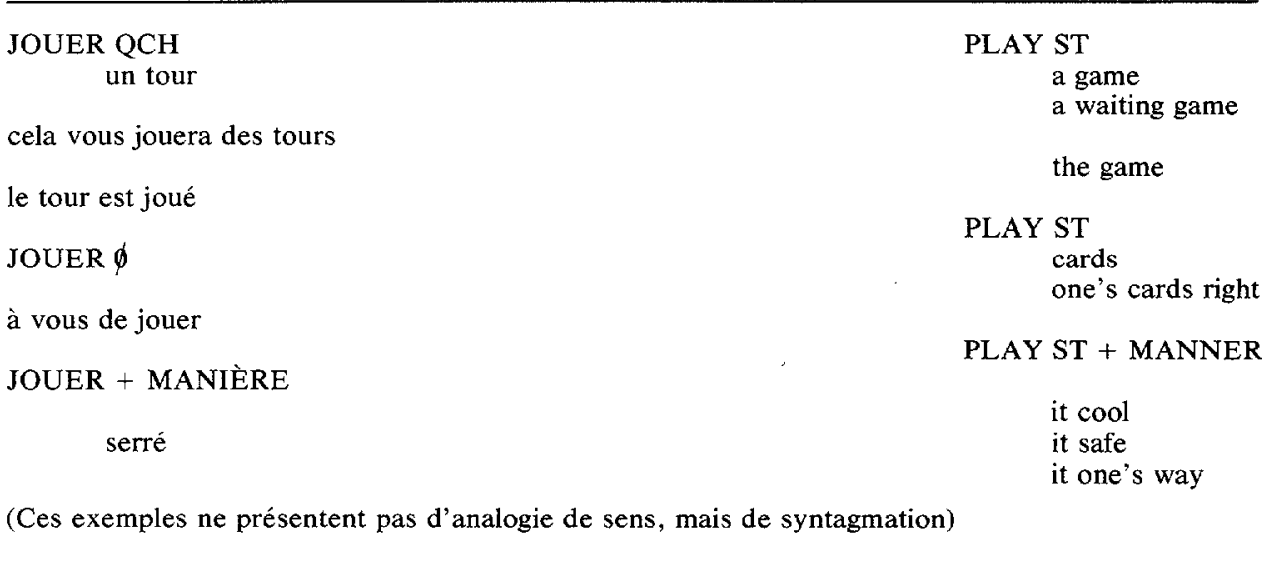

Cependant s'il y a analogie entre la fiche syntagmatique et l'entrée d'un dictionnaire récent (DFC, BOR, LEX), il ne s'agit pas pour le rédacteur de copier l'entrée en en modifiant seulement la disposition, mais à partir des consultations d'ordonner et de raisonner le développement sémantique sous forme de syntagmes.

Le traducteur disposant d'une fiche syntagmatique recoupant diverses sources possède ainsi un outil efficace et économique, en particulier dans le cas de termes neufs ou mal maîtrisés (l'exercice peut être correctif).

\section{Pour le traducteur}

La fiche syntagmatique, pour lui, ne présente en fait d'intérêt immédiat que si elle comporte non pas deux colonnes, mais trois ou quatre, encore que l'exercice de la paraphrase et de la synonymie (surtout rigoureuse et systématique et non simplement stylistique : pour éviter les répétitions) puisse lui assurer des moyens d'expression à la fois exacts et variés.

Bipartite, la fiche comportera d'un côté la construction suivie vers le bas d'un premier syntagme (position occupée), le syntagme peut être une phrase, comme dans qch battre : le tambour, le cœur, et de l'autre la paraphrase, c'està-dire sous une autre forme le sens de l'assemblage. Elle devrait cependant renvoyer à une fiche correspondante dans l'autre langue du traducteur.

L'idéal serait alors la fiche quadripartite, comportant un volet de plus que la fiche bilingue tripartite, volet de contrôle. 


\begin{tabular}{|c|c|c|c|}
\hline Fiche bipartite & construction $\rightarrow$ & paraphrase & \\
\hline Fiche tripartite & $\begin{array}{l}\text { construction } \rightarrow \\
\text { langue de départ }\end{array}$ & paraphrase $\rightarrow$ & $\begin{array}{l}\text { équivalent } \\
\text { langue d'arrivée }\end{array}$ \\
\hline Fiche quadripartite & $\begin{array}{l}\text { construction } \rightarrow \\
\text { 1. de dép. }\end{array}$ & $\begin{array}{l}\text { paraphrase } \rightarrow \\
\text { 1. de dép. }\end{array}$ & $\begin{array}{l}\text { équivalent } \rightarrow \text { paraphrase } \\
\text { 1. d'arr. } 1 . \text { d'arr. }\end{array}$ \\
\hline
\end{tabular}

TABLEAU II

\begin{tabular}{|c|c|c|c|}
\hline \multicolumn{4}{|c|}{ Première étape d'une fiche syntagmatique sur GROUND } \\
\hline $\begin{array}{l}\text { Mot-souche } \\
\text { et position } \\
\text { occupée }\end{array}$ & $\begin{array}{l}\text { paraphrase } \\
\text { anglaise }\end{array}$ & $\begin{array}{l}\text { Équivalence } \\
\text { française }\end{array}$ & $\begin{array}{l}\text { paraphrase } \\
\text { française }\end{array}$ \\
\hline \multicolumn{4}{|l|}{ GROUND ST } \\
\hline a ship & run ashore & échouer & $\begin{array}{l}\text { pousser à la côte } \\
\text { et immobiliser }\end{array}$ \\
\hline a plane & $\begin{array}{l}\text { keep on } \\
\text { ground }\end{array}$ & $\begin{array}{l}\text { retenir au } \\
\text { sol }\end{array}$ & $\begin{array}{l}\text { empêcher de se } \\
\text { mouvoir, immobiliser }\end{array}$ \\
\hline a pilot & $\begin{array}{l}\text { prevent } \\
\text { from } \\
\text { flying }\end{array}$ & $\begin{array}{l}\text { interdire } \\
\text { de voler }\end{array}$ & lui défendre de \\
\hline
\end{tabular}

Le volet de contrôle, on le comprend, permet de s'assurer que le dictionnaire bilingue ne se satisfait pas d'une approximation ou d'un rapport superficiel, car il faut bien le reconnaître, même le système synonymique utilisé par ROC n'est guère plus satisfaisant que la classique indication de domaine.

Il ne s'agit pas pour le traducteur de remplacer les dictionnaires mais de se constituer un moyen d'accès privilégié à ceux-ci, une méthode de lecture réduisant au minimum les aléas. Les fiches pourraient, toutefois, en nombre suffisant, présenter le modèle du dictionnaire (écrit ou automatisé) du traducteur futur.

\section{Préparation et rédaction}

Il faut tout d'abord se pénétrer du sémantisme du terme-souche des syntagmes à construire, par la consultation de plusieurs sources, des plus simples (West) aux plus élaborées ou complexes (LDC, SOD).

Ensuite, retenir les syntagmes à partir de ces mêmes sources, et les ordonner suivant l'occupation des positions ou l'adjonction de particules. S'ils sont répertoriés, le travail en est d'autant facilité, sinon les isoler en tronquant les phrases-exemples.

Dans les cas où on n'a pas pu recueillir de phrases-exemples (certaines sources sont peu généreuses (WEB)), on attendra les résultats de la fiche phraséologique, qu'il suffira alors de traiter comme phrase-exemple de dictionnaire. Comme le paraphrasage peut cependant présenter quelque difficulté, l'incertitude se marquera par un point d'interrogation. 
La disposition suivra les modèles ci-dessus et, en tête de la fiche, on placera le terme-souche, suivi de sa première construction, parfois absolue, et c'est alors la position sujet qui sera remplie. Pour l'ordre des constructions quand il s'agit de verbes, on pourra s'inspirer de Caput et Caput (1976).

On évitera de faire figurer des définitions au sens strict, qui trop souvent font intervenir des éléments encyclopédiques. Devant une définition ou une paraphrase trop longue, on procédera à une réduction, pour ne retenir que les éléments pertinents. Il y a sans doute là matière à un exercice de plus, proche de la contraction de texte.

Pour l'étudiant, il est bon de prévoir une fragmentation du temps consacré à la recherche et à la rédaction; ainsi pour éviter la saturation, on ne passera pas plus d'une heure sur chaque fiche.

\section{Fiche phraséologique}

La préparation de la fiche phraséologique fait appel à moins de systématisation, mais sollicite plus la sagacité du traducteur, tout en s'appuyant sur l'acquis du premier exercice.

Elle vise à familiariser son rédacteur avec ses outils quotidiens : dictionnaires, encyclopédies, manuels, ouvrages et revues spécialisés.

La recherche consiste à relever les «contextes » où apparaît un terme ou un syntagme: ces contextes seront en premier lieu les phrases-exemples des dictionnaires et les phrases descriptives des encyclopédies, qui fourniront les modèles de dépouillement ultérieur (seule l'accessibilité des sources constitue une limite).

Comme la fiche phraséologique doit assurer une connaissance élémentaire de la distribution d'un terme (ses collocations), elle devra présenter les environnements linguistiques les plus fréquents, c'est-à-dire donner une liste plus ou moins ordonnée d'énoncés dont les éléments sont plus ou moins attendus en fonction les uns des autres ${ }^{7}$.

Le dépouillement de textes spontanés peut être envisagé, mais on veillera auparavant à la bonne maîtrise de la méthode.

Dans le cas des langues de spécialité, la rédaction de la fiche devrait mener graduellement à une connaissance du domaine par le biais de la langue et fournir un dictionnaire portatif facilitant l'expression (et corollairement la traduction).

\section{Préparation et disposition}

Les étapes préalables sont les mêmes que pour la fiche syntagmatique: chercher à se pénétrer du sémantisme fondamental (entrées de plusieurs dictionnaires). Une fiche utile du point de vue phraséologique ne retient qu'un sens (acception) par fiche.

7. Sur la collocation et ses composants les collocables, voir Choul (1979). 
Le relevé devrait assurer entre 5 et 8 contextes pertinents - où des éléments cooccurrents différents opèrent la même sélection, constituant les variables d'une constante qu'est le sens.

Non seulement la fiche met-elle en évidence les affinités entre termes (et mots-outils, particules, etc.) mais elle permet également de répondre aux questions suivantes:

- que fait $\mathrm{x}$ (le terme)?

- que fait-on avec $x$ ?

- qu'est-ce qui caractérise $\mathrm{x}$ ?

- où?

- comment?

- pourquoi?

Un contexte complet comportera une combinaison maximale des réponses à ces questions.

La disposition en étoile semble la plus élégante et la plus efficace, ainsi haut fourneau présenterait entre autres les éléments phraséologiques suivants :

\section{TABLEAU III}

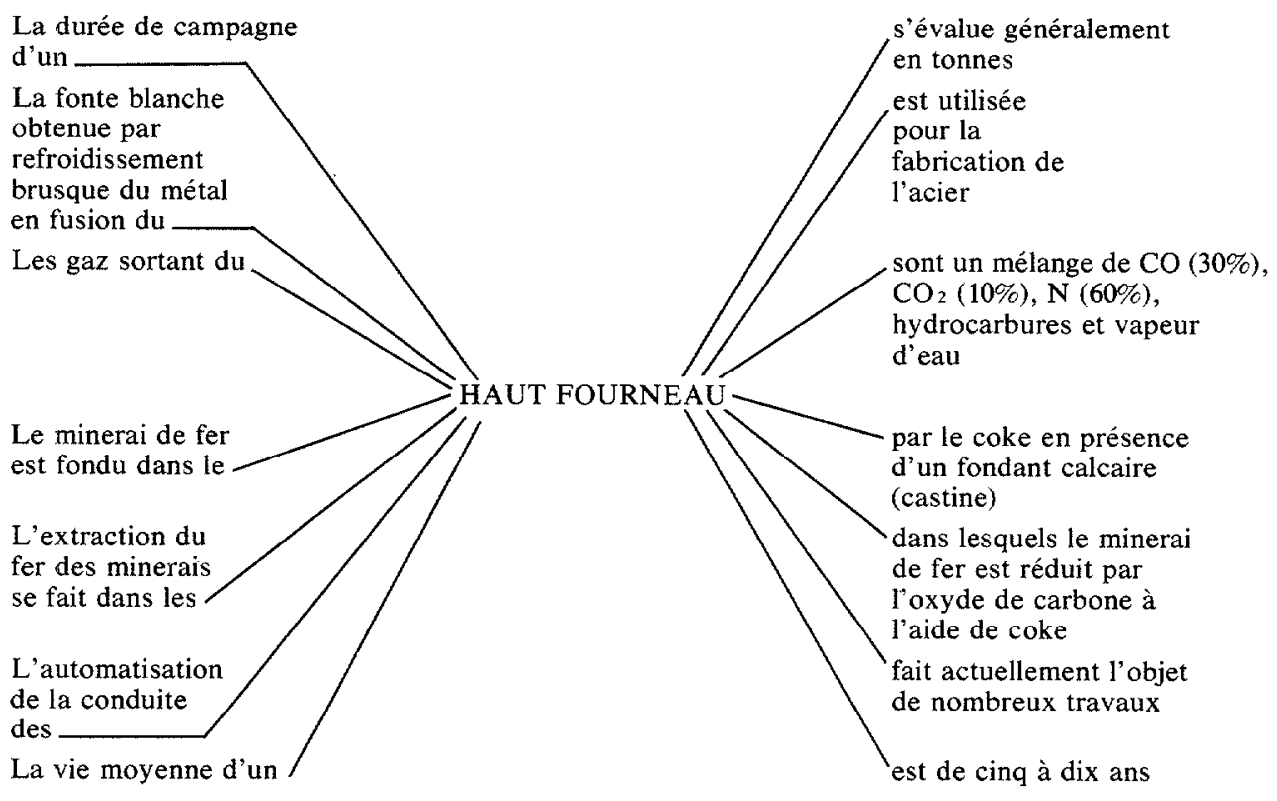

Comme pour la fiche syntagmatique, on évitera la fatigue et la saturation en ne consacrant qu'une heure à l'exercice. 


\section{Toujours les syntagmes}

Le troisième exercice utilise les mêmes données et présupposés théoriques, mais s'intéresse plus particulièrement (pour l'heure) aux syntagmes nominaux dont le degré de stabilisation varie et qui en anglais manifestent une grande unité morphologique généralement absente de leurs équivalents français.

L'exercice exploite particulièrement la notion de paraphrase, et pourrait permettre de résoudre les difficultés que l'on peut éprouver à paraphraser à partir des dictionnaires les noms composés et les unités terminologiques.

On distinguera trois étapes, suivies d'une série d'observations sur le comportement des unités sémantiques lors du passage d'une langue dans l'autre. On ajoutera éventuellement une étape d'abstraction qui fournirait la relation sémantique fondamentale justifiant la collocation des deux termes.

L'exercice se fonde sur le relevé provisoire de Dillon (1977, 50-52), disposé en tableau ci-dessous:

TABLEAU IV

\begin{tabular}{|c|c|c|c|}
\hline $\begin{array}{l}\text { lawn mower } \\
\text { can opener } \\
\text { hedge clippers }\end{array}$ & what one uses to $\mathrm{VN}$ & $\begin{array}{l}\text { ash tray } \\
\text { flower box } \\
\text { shoe polish }\end{array}$ & $\begin{array}{l}\mathrm{N} \text { put on/in } \mathrm{N} \\
\text { (either order) }\end{array}$ \\
\hline $\begin{array}{l}\text { wood cutter } \\
\text { house painter } \\
\text { truck driver }\end{array}$ & one who VsN & $\begin{array}{l}\text { candle light } \\
\text { fever blister } \\
\text { water pistol }\end{array}$ & $\mathrm{N}$ gives/produces $\mathrm{N}$ \\
\hline $\begin{array}{l}\text { food poisoning } \\
\text { sun burn } \\
\text { storm damage }\end{array}$ & $\mathrm{N}_{2}$ caused by $\mathrm{N}_{1}$ & $\begin{array}{l}\text { oil well } \\
\text { coal mine } \\
\text { silk worm }\end{array}$ & $\mathrm{N}_{1}$ taken/obtained from $\mathrm{N}_{2}$ \\
\hline $\begin{array}{l}\text { steam cleaning } \\
\text { gun fight } \\
\text { sliderule calculation }\end{array}$ & $\mathrm{N}_{2}$ done with $\mathrm{N}_{1}$ & $\begin{array}{l}\text { apple sauce } \\
\text { grape wine } \\
\text { peanut butter }\end{array}$ & $N_{2}$ made from $N_{1}$ \\
\hline $\begin{array}{l}\text { heart failure } \\
\text { population growth } \\
\text { earthquake }\end{array}$ & $\mathrm{N}_{2}$ of $\mathrm{N}_{1}$ & $\begin{array}{l}\text { alcohol lamp } \\
\text { air rifle } \\
\text { windmill }\end{array}$ & $\mathrm{N}_{2}$ powered by $\mathrm{N}_{1}$ \\
\hline $\begin{array}{l}\text { home cooking } \\
\text { boat ride } \\
\text { room service }\end{array}$ & $\mathrm{N}_{2}$ at/on/it $\mathrm{N}_{1}$ & $\begin{array}{l}\text { bug spray } \\
\text { gas mask } \\
\text { heat shield }\end{array}$ & $\mathrm{N}_{2}$ to keep away $\mathrm{N}_{1}$ \\
\hline $\begin{array}{l}\text { retaining wall } \\
\text { scrub woman } \\
\text { demolition squad }\end{array}$ & $\mathrm{N}_{2}$ that $\mathrm{Vs}$ & $\begin{array}{l}\text { life boat } \\
\text { cash box } \\
\text { safety belt }\end{array}$ & $\mathrm{N}_{2}$ to keep/retain/preserve $\mathrm{N}_{1}$ \\
\hline $\begin{array}{l}\text { washing machine } \\
\text { drinking cup } \\
\text { playing card }\end{array}$ & $\mathrm{N}_{2}$ that one uses to $\mathrm{V}$ & $\begin{array}{l}\text { mailman } \\
\text { milk truck } \\
\text { oil pipe }\end{array}$ & $\mathrm{N}_{2}$ carries/conveys $\mathrm{N}_{1}$ \\
\hline $\begin{array}{l}\text { asking price } \\
\text { smelling salts } \\
\text { push button }\end{array}$ & $\mathrm{N}_{2}$ that is Ved & $\begin{array}{l}\text { dragontly } \\
\text { kettledrum }\end{array}$ & $\mathrm{N}_{2}$ resembles $\mathrm{N}_{1}$ \\
\hline $\begin{array}{l}\text { swimming pool } \\
\text { playground } \\
\text { storage battery }\end{array}$ & \multicolumn{3}{|l|}{$\mathrm{N}_{2}$ where one $\mathrm{Vs}$} \\
\hline $\begin{array}{l}\text { (On trouvera dans } \\
\text { plus longues listes d }\end{array}$ & $\begin{array}{l}\text { ms (1976) un classem } \\
\text { mples) }\end{array}$ & différent, moin & émantique, mais également de \\
\hline
\end{tabular}




\section{Micro-traduction}

Armé de ce tableau comme moyen de contrôle, on tentera de paraphraser, de classer et d'ordonner des syntagmes binominaux (binomials, compound nouns, noun compounds) recueillis lors de la consultation de dictionnaires ou au cours de lectures. Ainsi cowcatcher, cable-ship, deux exemples particulièrement difficiles, seront d'abord paraphrasés en anglais, et l'on tentera de les rapprocher des catégories isolées par Dillon (à partir de travaux d'autres auteurs).

En classe, les séries de Dillon peuvent servir d'exercice de découverte : à partir du principe que les éléments présents partagent une continuité de relation, on essaie par approximations successives de reconnaître la paraphrase unique.

La deuxième étape consiste à traduire la paraphrase structurelle du syntagme, dont le résultat pourra être remanié après la troisième étape, par exemple:

\section{$N_{2}$ caused by $N_{1} \rightarrow N$ causé par $N$}

C'est avec la traduction proprement dite que l'on pourra procéder aux observations. Si le résultat du paraphrasage de cowcatcher est $/ \mathrm{N}_{2}$ to keep $\mathrm{N}_{1}$ away/ le passage vers chasse-pierres (l'équivalent français) est facilité par l'étape intermédiaire : / $\mathrm{N}_{1}$ pour chasser $\mathrm{N}_{2} /$ qui comporte cependant une transformation en $\mathrm{V}+\mathrm{N}$, sur le modèle propre au français chasse-neige, chassemouches, chasse-roue.

Le paraphrasage intralinguistique de cable-ship ne pourra cependant se faire d'emblée en $/ \mathrm{N}_{2}$ that lays $\mathrm{N}_{1} /$, en particulier si l'on cherche un modèle chez Dillon ou dans une de ses sources (Adams 1976), car il s'agit d'une apposition et la paraphrase structurelle devra s'inspirer d'une définition: a ship that lays cable, et la paraphrase française subira encore une transformation: $\mathrm{N}_{1}$ qui pose $\mathrm{N}_{2} \rightarrow \mathrm{N}$. Il s'agit en effet de câblier, obéissant au modèle très productif de minerai $\rightarrow$ minéralier, céréales $\rightarrow$ céréalier, pétrole $\rightarrow$ pétrolier, méthane $\rightarrow$ méthanier, bananes $\rightarrow$ bananier, etc. Les observations pourront faire ressortir un des premiers exemples: négrier, mais aussi qu'une paraphrase de Dillon convenait, en réalité, puisqu'en français câblier est associé à $\mathrm{N}_{2}$ carries/conveys $\mathrm{N}_{1}$ (oil pipe, et que l'on songe à (oil tanker, ore carrier, etc.).

On voit que cowcatcher et cable-ship diffèrent considérablement de sunburn et silk-worm (voir le tableau) qui en français deviennent des synapsies, l'une et l'autre très stabilisées ${ }^{8}$. Les observations porteront donc sur la multiplicité des formes en français (coup de soleil, ver à soie, intoxication alimentaire, État tampon, voiture-restaurant, etc.) et pourront s'inspirer de ces quelques questions: dans le passage de l'anglais vers le français, faut-il réordonner le syntagme? est-il maintenu (mailman $\rightarrow$ facteur) ? y a-t-il transformation grammaticale $(\mathrm{N}+\mathrm{N} \rightarrow \mathrm{N}+\mathbf{A d j}$, etc.) ? y a-t-il insertion de particule? la cohésion sémantique est-elle suffisante pour que l'on puisse parler de synapsie, de lexie complexe ou même de nom composé? Rappelons que l'opacification de coup

8. Voir Benveniste (1976). 
de soleil (où coup = brûlure et de = causé par le) est un indice de stabilisation que marque encore l'impossibilité de manipulation en *à coups de soleil, sur le modèle de à coups de canne. L'opacification est nette en français avec marée noire tandis que son équivalent anglais oil spill n'est opaque qu'en partie, mais l'un comme l'autre ne sont pas d'emblée paraphrasables si l'on s'en tient aux catégories de Dillon: à moins que l'on consente à voir la catégorie $/ \mathrm{N}_{2}$ resembles $\mathrm{N}_{1} /$, mais ce ne serait valable que pour marée noire et encore après transformation $\left(\mathrm{N}_{1}\right.$ resembles $\left.\mathrm{Adj}\right)$. Sous la contrainte extra-linguistique on pourra songer à une nouvelle catégorie / $N$ qui pollue/ où l'on pourrait ranger pluie acide, tandis que pour l'anglais on devra se contenter d'une variante de $\mathrm{N}_{2}$ that is $V e d \rightarrow \mathrm{N}_{1}$ that is Ved.

Ces anomalies tendent sans doute à montrer l'insuffisance de la paraphrase structurelle proposée par Adams 1976 et Dillon 1977, mais cela tient sans doute à ce qu'elle forme un compromis entre la paraphrase de sens (acception) et la relation fondamentale (food poisoning $\rightarrow$ causation). L'exercice peut donc se poursuivre avec la recherche de cette relation, de type sémiotique, et non plus sémantique. On peut en proposer un premier tableau provisoire, issu de recoupements (L3, DFC, Vigner et Martin 1976, Benveniste 1976).

TABLEAU $V$

\begin{tabular}{|c|c|}
\hline RELATION & EXEMPLES \\
\hline appartenance & $\begin{array}{l}\text { un ami à moi, une manière à soi, l'organe porte-pièce du tour, le } \\
\text { bouchon de la pompe }\end{array}$ \\
\hline $\begin{array}{l}\text { partie à } \\
\text { tout }\end{array}$ & verre de montre, pied de table, peau de porc \\
\hline $\begin{array}{l}\text { classe } \\
\text { d'individus }\end{array}$ & robe d'avocat, béret de matelot, voiture d'enfant \\
\hline $\begin{array}{l}\text { destination } \\
\text { (usage) }\end{array}$ & $\begin{array}{l}\text { tasse à thé, verre à boire, vase à fleurs, salle de spectacle, brosse à } \\
\text { habit, vis de blocage, abrasif aggloméré pour tonnelage }\end{array}$ \\
\hline manière & marche au pas, photographie de face, chambre avec vue sur le jardin \\
\hline $\begin{array}{l}\text { moyen } \\
\text { (instrument) }\end{array}$ & $\begin{array}{l}\text { machine à vapeur, chauffage au gaz, coup de pied, envoi par la poste, } \\
\text { lustrage avec un chiffon de laine }\end{array}$ \\
\hline agent & $\begin{array}{l}\text { incorruptibilité à l'humidité, écrasement de l'armée par des forces plus } \\
\text { importantes }\end{array}$ \\
\hline $\begin{array}{l}\text { agent moteur } \\
\text { (cf. moyen) }\end{array}$ & moulin à vent, avion à réaction, lampe à pétrole \\
\hline $\begin{array}{l}\text { objet de } \\
\text { l'action }\end{array}$ & nettoyage de la buse, contrôle du débit, flexion de l'outil-couteau \\
\hline $\begin{array}{l}\text { fonction } \\
\text { (cf. destination) }\end{array}$ & raccord d'aspiration, pompe d'injection, écrou de blocage \\
\hline cause & la surprise de cette arrivéc, larmes de joie \\
\hline matière & $\begin{array}{l}\text { collier d'or, maison de brique, table en bois, statue en bronze, pla- } \\
\text { quette de carbure }\end{array}$ \\
\hline
\end{tabular}




\begin{tabular}{ll} 
caractérisation & $\begin{array}{l}\text { navire à l'ancre, appareil à commande manuelle, vis à tête fendue, } \\
\text { transports par eau, gravure sur verre }\end{array}$ \\
\hline $\begin{array}{l}\text { caractéristique } \\
\text { distinctive }\end{array}$ & $\begin{array}{l}\text { bête à cornes, équerre à chapeau, pompe avec amorçage à main, } \\
\text { niveau à cadre }\end{array}$ \\
\hline $\begin{array}{l}\text { temps } \\
\text { (durée, date) }\end{array}$ & départ à l'aube, bons à cinq ans, débarquement de nuit \\
\hline terme & retour pour dimanche \\
\hline simultanéité & le lever avec le jour \\
\hline $\begin{array}{l}\text { circonstance } \\
\text { lieu }\end{array}$ & chemise de nuit, table de travail, salle de jeux, fusil de chasse \\
\begin{tabular}{ll} 
provenance) & son passage à Lyon, eau de source \\
\hline direction & départ pour l'Espagne \\
\hline $\begin{array}{l}\text { réciprocité } \\
\text { (échange, } \\
\text { comparaison) }\end{array}$ & jour pour jour, envoi contre remboursement \\
\hline $\begin{array}{l}\text { opposition } \\
\text { (contraste) }\end{array}$ & combat avec un ennemi supérieur en nombre, envers et contre tous \\
\hline $\begin{array}{l}\text { accompagnement } \\
\text { (accord, réunion) }\end{array}$ & promenade avec ses enfants \\
\hline $\begin{array}{l}\text { dimension } \\
\text { (proportion, répétition) }\end{array}$ & trois mètres sur quatre, deux jours sur huit, sottises sur sottises \\
\hline $\begin{array}{l}\text { apposition } \\
\text { métaphore }\end{array}$ & ville de Paris, axe de rotation \\
\hline & tête de loup, pied de biche, dent de lion \\
\hline
\end{tabular} \\
\hline
\end{tabular}

Il est clair qu'avec cet outil même imparfait on ne peut plus se satisfaire de la caractérisation comme relation fourre-tout (cf. Grevisse qui fait de par eau, sur verre, de marbre des caractérisations).

Le tableau gagnerait à être réordonné puisqu'on note qu'un certain nombre de relations sont plutôt des spécialisations d'autres, comme/circonstance/ (cf. Benveniste) l'est de /temps/, de même que /terme/. Les difficultés tiennent non pas à la méthode ou à l'exercice, mais bien à l'obsession du classement, et l'on peut noter que Adams $(1976,69)$ placera la fonction comme sous-relation de l'apposition avec comme exemples sister ship, fuel oil, nose cone (dont la paraphrase structurelle serait : $B$ has the function of $A$ ), ce qui est discutable avant même que l'on tente de traduire (navire-jumeau, mazout, pointe ou coiffe ou encore revêtement de protection). L'indécision dans l'équivalence de nose cone en français (cf. BT 155) marque à la fois la difficulté que l'on a à justifier une relation quelconque et à paraphraser c'est-à-dire à assigner un sens. Pourquoi ne pas retenir nez ou ogive (cf. PEL) quitte à le syntagmer avec sa caractéristique ou sa fonction : à bouclier thermique, de protection (cf. LID), ou encore, comme nose cone semble être construit sur nose piece, pourquoi ne pas retenir nasal, comme pour l'armure (le nose-cone protège le nose)? 
On le voit, la recherche des relations, tout comme le paraphrasage, permet un certain nombre de spéculations non dénuées d'intérêt, en particulier pour la terminologie.

Ce qu'il faut souligner c'est qu'un exercice de ce genre permet d'éclater les listes bilingues trop souvent compilées au petit bonheur la chance, et de raisonner les dénominations que l'on considère à tort comme une propriété des choses.

L'exercice s'est limité jusqu'à présent aux syntagmes à deux noms à fonction nominale (noun compounds), mais on pourra l'étendre aux composés adjectivaux (adjective compounds) également caractéristiques de l'anglais (coolheaded, clean-shaven, dog-tired) dont les équivalents laissent présager d'intéressants développements intermédiaires : de sang-froid ou imperturbable, glabre ou rasé de frais, éreinté ou mort de fatigue, etc.

On peut aussi considérer l'exercice sur les paraphrases structurelles ou sémantiques (les deux sont possibles) et les relations comme un premier pas (débroussaillement) vers l'intégration des noms composés et syntagmes nominaux à la fiche syntagmatique qui privilégie pour l'instant les verbes et leurs compléments (ainsi que leurs sujets, dans les constructions absolues en particulier).

La résistance des lexies composées ou complexes à l'intégration à la fiche syntagmatique tient en effet à ce que la définition lexicographique tend encore toujours à décrire l'objet (définition de type encyclopédique) plutôt que les relations entre signifiés.

\section{Pour le traducteur}

Même si dans une certaine mesure il a pu sembler que nous nous écartions de la traduction par des spéculations digressives, nous n'avons jamais vraiment perdu de vue l'objet des exercices : élargir la perspective du manipulateur de signes qu'est le traducteur, et montrer combien est trompeuse la flèche (LD $\rightarrow$ LA) reliant la langue de départ à la langue d'arrivée.

Si l'observation du comportement extérieur du traducteur en exercice invitait à la caricature, les exercices dépassent le simple jeu de mots pour donner une idée de la complexité de sa tâche, en lui proposant de la maximiser.

Moyens d'apprentissage, outils de travail, délassement (pourquoi pas?), ces exercices invitent à la discussion que l'on souhaitera nourrie et profitable ${ }^{9}$.

\section{BIBLIOGRAPHIE}

ADAMS, V., 1976, An Introduction to Modern English Word-formation, London, Longman. BENVENISTE, E., 1976, Problèmes de linguistique générale II, Paris, Gallimard.

9. Elle a déjà commencé dans les classes de version technique et générale de $3^{\mathrm{e}}$ année et de version spécialisée de $4^{e}$ année à l'E.T.I. de l'Université d'Ottawa, et je remercie mes étudiants de leur active participation. 
BOR, 1976 - DAVAU, M. et al., Dictionnaire du frunçais vivant, Paris, Bordas.

BT 155, 1977 - CANADA, Bureau des traductions, Direction générale de la terminologie et de la documentation, Arrêtés de terminologie de la République française (Bulletin de terminologie 155), Ottawa.

CAPUT, J. et J.-P., 1976, Dictionnaire des verbes français, Paris, Larousse.

CHOUL, J.-C., 1979, «La méthode du discours: quelques outils», Meta 24/3, Montréal, Presses de l'Université de Montréal, sept. 1979, p. 336-348.

DDL, 1976 - GALISSON, R. et D. COSTE, Dictionnaire de didactique des langues, Paris, Gallimard (coll. " $F$ ").

DFC, 1971 - DUBOIS, Jean et al., Dictionnaire du français contemporain, Paris, Larousse.

DILLON, G. L., 1977, Introduction to Contemporary Linguistic Semantics, Englewood Cliffs (N.J.), Prentice-Hall.

DUBUC, R., 1978, Manuel pratique de terminologie, Montréal/Paris, Linguatech/C.I.L.F.

GREIMAS, A. J. et J. COURTES, 1979, Sémiotique, dictionnaire raisonné de la théorie du langage, Paris, Hachette.

GREVISSE, M., 1964, Le Bon Usage, $8^{\mathrm{e}}$ éd. revue, $3^{\mathrm{e}}$ tirage, Gembloux, Duculot.

GUIRAUD, P., 1970, Structures étymologiques du lexique français, Paris, Larousse.

L3, 1965, Larousse trois volumes en couleurs, Paris, Larousse.

LDC, 1978, - PROCTOR, P. (éd.), Longman Dictionary of Contemporary English, London, Longman.

LE GOFFIC, P. et N. COMBE McBRIDE, 1975, Les Constructions fondamentales du français, Paris, Hachette/Larousse (coll. Le français dans le monde-B.E.L.C.).

LEX, 1977 - Larousse de la langue française, Lexis, Paris, Larousse.

LID, 1972 - Larousse Illustrated International Encyclopedia and Dictionary, s.1., Larousse/ McGraw Hill.

LYONS, J., 1977, Semantics, Cambridge, Cambridge University Press.

PEL, 1975 - Petit Larousse illustré, Paris, Larousse.

ROC, 1978 - Robert-Collins dictionnaire français-anglais, anglais-français, Paris, Société du Nouveau Littré.

SOD, 1973 - ONIONS, C. T. (ed.), The shorter Oxford English Dictionary, $3^{\mathrm{e}}$ éd., Oxford, Clarendon Press.

VIGNER, G. et A. MARTIN, 1976, Le Français technique, Paris, Hachette/Larousse (coll. Le français dans le monde-B.E.L.C.).

WEB, 1970 - Webster's Seventh New Collegiate Dictionary, Toronto, Th. Allen \& Son.

WEST, M., 1961, The New Method Dictionary, London, Longman. 